\title{
Essai de caractérisation de quelques lacs rwandais par leur peuplement piscicole
}

\author{
C. K. Kanangire ${ }^{1}$ \\ E. Depiereux ${ }^{2}$ \\ J.-C. Micha ${ }^{2}$
}

Mots-clés : Rwanda, lacs, caractérisation, peuplement piscicole.

Dix lacs du Rwanda ont été caractérisés à partir des résultats de nombreuses pêches expérimentales standardisées. L'analyse en composantes principales et l'analyse de regroupement appliquées sur les captures des espèces de poissons montrent que les différentes espèces se rangent le long d'un continuum sur une structure curvilinéaire indiquant une augmentation du nombre d'espèces d'amont en aval du réseau fluviatile Nyabarongo-Akagera. La classification des lacs montre l'existence de trois groupes : les lacs d'altitude, pauvres en espèces, les lacs de la vallée de la Nyabarongo, moins pauvres, et les lacs de la dépression de l'Akagera, à plus grande biodiversité. Cette disposition des peuplements de poissons et des lacs montre l'importance de la rivière Nyabarongo-Akagera dans l'écologie des écosystèmes lentiques rwandais.

\section{Characterization of some Rwandan lakes based on their fish populations}

Keywords : Rwanda, lakes, characterization, fish populations.

Ten Rwandan lakes have been characterized on the basis of two year's of fisheries data. Principal component analysis and cluster analysis applied to fish data show that different species are arranged on a curvilinear structure and that their number increases from upsteam to downstream in the Nyabarongo-Akagera river. The classification of the lakes shows three groups : the northern poor lakes, the less poor Nyabarongo valley lakes and the Akagera valley lakes with the highest variety of fish species. This pattern shows the role of the Nyabarongo-Akagera river in the ecology of Rwandan aquatic ecosystems.

\section{Introduction}

Le Rwanda dispose d'un important réseau aquatique dont les lacs occupent environ $1250 \mathrm{~km}^{2}$, soit $4,7 \%$ de la superficie totale du pays.

Les premières études des lacs rwandais faites par Damas (1953, 1954 a, 1954 b, 1955 et 1956) donnent des informations qui servent toujours de référence pour la limnologie des lacs rwandais. Quelques travaux ont suivi ceux de Damas : Kiss (1976 et 1977), Plisnier et al. (1988), Plisnier (1990), Mukankomeje

1. Université Nationale du Rwanda, Fac. des Sciences, B.P. 629 Butare, Rwanda.

2. Facultés Universitaires N.-D. de la Paix, Rue de Bruxelles 61, B5000 Namur, Belgique.
(1992), Mukankomeje et al. (1993) ainsi que l'inventaire à grande échelle réalisé par le Bureau National d'Etudes et Programmes (BUNEP, 1988). Après un traitement global des données réunies au cours de l'étude BUNEP par Kanangire (1988), les données piscicoles ont été publiées par Micha \& Frank (1992) et celles relatives aux algues par Descy (1995). Cependant la caractérisation des peuplements de poissons des lacs rwandais est encore embryonnaire et ce travail vise à combler cette lacune.

\section{Matériel et méthodes}

Dans les 10 lacs étudiés, une batterie de filets standards expérimentaux, construits avec 10 nappes à mailles de 8 à $60 \mathrm{~mm}$ d'entre-nœuds, de $2 \mathrm{~m}$ de chute sur $25 \mathrm{~m}$ de longueur et reliés les uns aux autres; ont été systématiquement utilisés à la fois en zone pélagique et en zone côtière. 
Les pêches ont été programmées de manière à couvrir les quatre saisons typiques au Rwanda. Les filets ont été posés tous les soirs vers $18 \mathrm{~h}$ et relevés tous les matins vers $6 \mathrm{~h}$. Le démaillage des poissons était effectué sur la terre ferme. Les poissons capturés dans chaque panneau étaient identifiés, comptés et pesés (Micha \& Frank 1992). Le tableau 2 présente les taxons rencontrés dans chaque lac ainsi que leur abondance.

Après transformation logarithmique $(\ln (x+1))$, ces données ont subi un traitement par analyse en composantes principales (ACP) et de regroupement des lacs (cluster analysis) (Lebart et al. 1979, Legendre \& Legendre 1979, Depiereux et al. 1983, Depiereux \& Feytmans 1985) en utilisant le logiciel STATISTICA (Statsoft 1994).

\section{Cadre géographique}

Les lacs concernés sont situés dans le réseau Nyabarongo-Akagera. Il s'agit des lacs Bulera (alt. $1862 \mathrm{~m}$ ) et Ruhondo (alt. $1764 \mathrm{~m}$ ), au nord; du lac Muhazi (alt. $1450 \mathrm{~m}$ ), au centre du pays ; des lacs Mugesera (alt. $1374 \mathrm{~m}$ ), Sake (alt. $1350 \mathrm{~m}$ ) et Bilira (alt. $1372 \mathrm{~m}$ ), de la dépression du Bugesera et des lacs Rwampanga, Cyambwe, Nasho et Thema de la plaine de l'Akagera (alt. $1290 \mathrm{~m}$ ). Ces lacs (Fig. 1) sont décrits par Micha \& Frank (1992).

La plupart de ces lacs sont situés dans la plaine d'inondation de l'Akagera et de la Nyabarongo, et certains d'entre eux sont en connexion étroite permanente avec celle-ci. Ainsi, les lacs Rwampanga et Nasho sont en communication avec l'Akagera par des canaux alors que les lacs du Bugesera sont séparés de la Nyabarongo par des marais à papyrus (BUNEP, 1988).

\section{Lacs étudiés}

\subsection{Caractéristiques physiques et chimiques}

Les mesures effectuées par BUNEP (BUNEP, 1988) concernent de nombreux paramètres permettant de caractériser ces lacs, tant d'un point de vue morphologique que de la qualité de leurs eaux. Toutefois si les mesures de concentration en anions et cations principaux sont satisfaisantes, la précision des teneurs en nitrates et orthophosphates, toujours en faible concentration, n'est pas suffisante et ces données ne seront pas utilisées dans le cadre de ce travail.

Après sélection des diverses données, les valeurs suivantes (Tableau 1) peuvent être retenues pour caractériser les lacs rwandais (Kanangire 1988, Descy 1995).
L'examen des paramètres physiques permet de distinguer parmi les dix lacs étudiés deux catégories nettes : les lacs profonds d'altitude - Bulera et Ruhondo - et les lacs peu profonds d'altitude moyenne : ceux du Bugesera - Mugesera, Bilira et Sake - et de l'Akagera -Rwampanga, Nasho, Cyambwe et Ihema-. Au point de vue chimie des eaux, les lacs Bulera et Ruhondo sont peu minéralisés de même que les lacs peu profonds de la dépression du Bugesera et de la vallée de l'Akagera. Le lac Muhazi constitue un cas à part. Il est peu profond, fort allongé et nettement plus minéralisé.

\subsection{Phytoplancton}

Comme le montrent Kanangire (1988) et Descy (1995), les lacs étudiés peuvent être classés en trois catégories selon le phytoplancton.

Les lacs peu profonds du Bugesera et de l'Akagera, présentent un phytoplancton peu variable dans le temps et dominé par des Cyanobactéries à très petites cellules ou à filaments minces (Microcystis holsatica Lemm., M. elachista (W. \& G. S. West) Compere, $M e$ rismopedia punctata Mey, Limnothrix limnetica Lemm.) accompagnées de nombreux taxons de Chlorococcales. Les diatomées y sont rarement bien représentées.

Parmi les lacs peu profonds, les lacs Rwampanga et Nasho se singularisent par l'abondance des diatomées, en particulier Eunotia zasuminensis (Cabejsz.) Korner, Synedra acus Kütz., Aulacoseira ambigua (Grun.) O. Müll.; A. granulata (Ehr.) Ralfs et var. Angustissima O. Müll. ainsi que des desmidiées, alors que les Cyanobactéries y sont nettement moins représentées.

Le lac Bulera se distingue par la faible représentation des Cyanobactéries et des Chlorococcales et par une relativement grande diversité des diatomées des genres Cyclotella, Aulacoseira et Synedra. Ces diatomées et la Dinophycée Ceratium hirundinella y sont plus abondantes en période de stratification. Le phytoplancton du lac Ruhondo occupe une position intermédiaire entre le Bulera et les lacs peu profonds et présente une composition algale variable. Il serait fort marqué par la saisonnalité (Descy 1995).

\section{Résultats : les peuplements de poissons}

Les résultats d'analyse de regroupement sont présentés sous la forme d'un dendrogramme (Fig. 2) montrant l'existence de trois groupes de lacs: les lacs Bulera, Ruhondo et Muhazi, les lacs Mugesera, Sake, Bilira et Nasho et les lacs Rwampanga, Cyambwe et thema. 


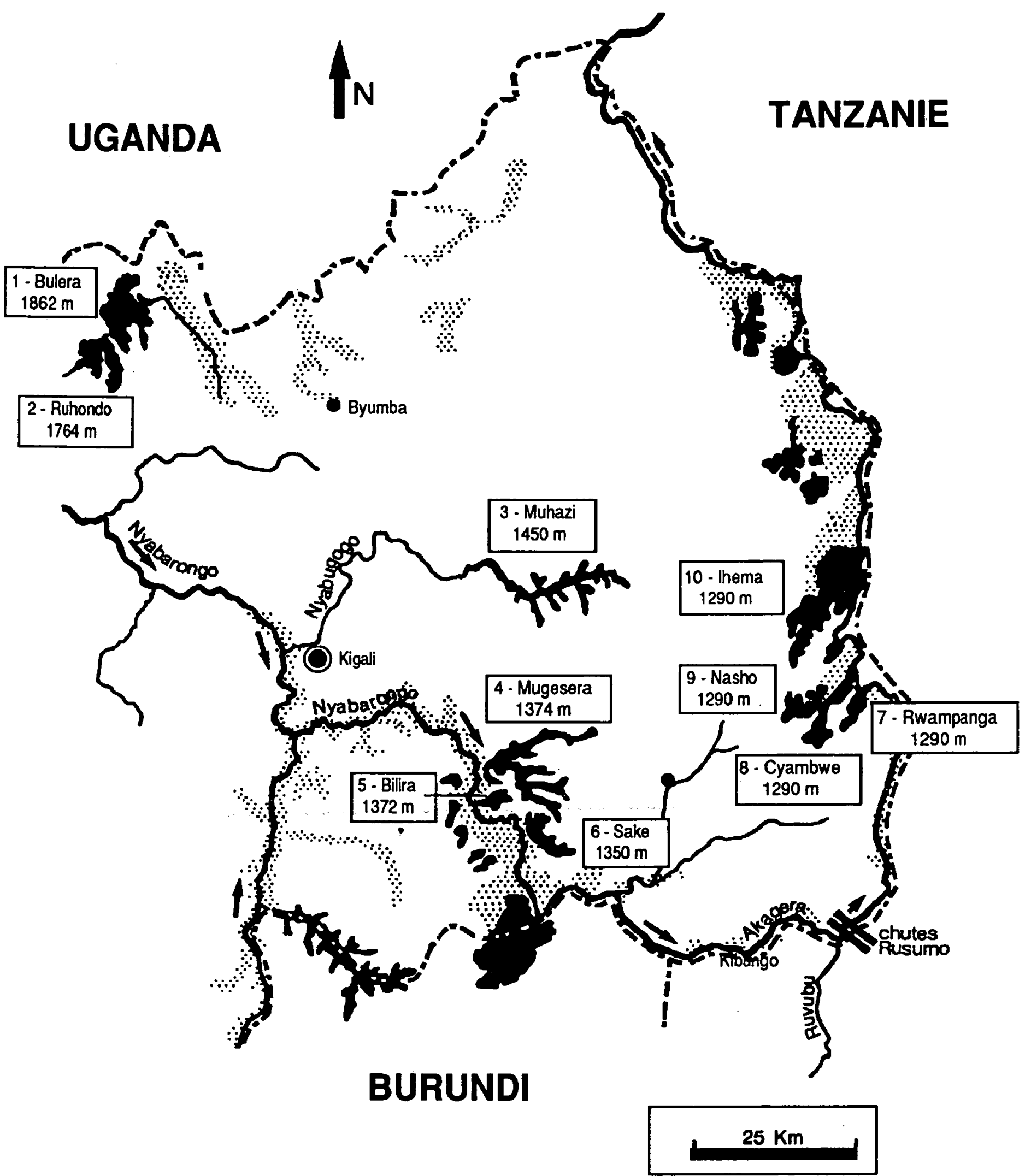

\begin{tabular}{|cccccc|}
\hline-1 & frontières & Butare & chefs-lieux de préfecture & Akagera & cours d'eau \\
\hdashline & 0 & Kigali & capitale & 0 & lacs ( $n^{\circ}+$ altitude) \\
\hline
\end{tabular}

Fig. 1. Localisation des lacs étudiés au Rwanda.

Fig. 1. Location of lakes studied in Rwanda . 
Tableau 1. Caractéristiques physiques et chimiques des dix lacs rwandais étudiés.

$\mathrm{Zm}$ : profondeur maximale en $\mathrm{m}, \bar{Z}$ : profondeur moyenne en $\mathrm{m}, \mathrm{Zs}:$ profondeur de disparition du disque de Secchi en $\mathrm{m}, \mathrm{Zeu}$ : épaisseur de la zone euphotique en $\mathrm{m}$, Cond : conductivité à $25^{\circ} \mathrm{C}$ en uS/cm, TAC : titre alcalimétrique complet, Ca++ : concentration en calcium (mg/).

Table 1. Physical and chemical characteristics of the ten rwandan lakes.

$\mathrm{Zm}$ : maximum depth, $\bar{Z}$ :mean depth, $Z s$ : transparency (Secchi disk), Zeu : depth of euphotic zone, Cond : conductivity at $25^{\circ} \mathrm{C}$, $T A C$ : Total alkalinity, $\mathrm{Ca}++$ : calcium concentration $(\mathrm{mg} / \mathrm{l})$

\begin{tabular}{|c|c|c|c|c|c|c|c|c|c|c|}
\hline & $\begin{array}{c}\text { Altitude } \\
\text { m }\end{array}$ & $\begin{array}{c}\text { Superficie } \\
\mathrm{km}^{2}\end{array}$ & $\begin{array}{c}\mathrm{Zm} \\
\mathbf{m}\end{array}$ & $\begin{array}{l}\bar{Z} \\
\mathrm{~m} \\
\end{array}$ & $\begin{array}{c}\mathrm{Zs} \\
\mathrm{m}\end{array}$ & $\begin{array}{c}\text { Zeu } \\
\text { m }\end{array}$ & $\begin{array}{c}\text { Cond.K25 } \\
\mu \mathrm{S} / \mathrm{cm}\end{array}$ & $\mathrm{pH}$ & $\begin{array}{c}\text { TAC } \\
\text { méq } ~^{-1}\end{array}$ & $\begin{array}{l}\mathrm{Ca}^{++} \\
\mathrm{mg} \mathrm{l}^{-1}\end{array}$ \\
\hline L. Bulera & 1862 & 55 & 173 & 100 & 4.9 & 11.3 & 115 & 7.4 & 1.1 & 5.8 \\
\hline L. Ruhondo & 1764 & 28 & 68 & 40 & 1.0 & 2.3 & 258 & 8.0 & 1.1 & 16.3 \\
\hline L. Muhazi & 1443 & 34 & 13 & 10 & 0.7 & 1.5 & 533 & 7.9 & 2.3 & 61.2 \\
\hline L. Mugesera & 1374 & 54 & 3.8 & 3 & 0.4 & 0.8 & 284 & 8.2 & 1.2 & 14.9 \\
\hline L. Bilira & 1350 & 6 & 6.5 & 5.5 & 0.5 & 1.1 & 191 & 7.7 & 1.4 & 11.1 \\
\hline L. Sake & 1350 & 20 & 4.3 & 4 & 0.7 & 1.5 & 160 & 8.4 & 0.9 & 12.2 \\
\hline L. Nasho & 1300 & 13.5 & 6.3 & 4.2 & 0.6 & 1.3 & 325 & 7.5 & 2.0 & 16.4 \\
\hline L. Rwampanga & 1300 & 9.5 & 7 & 5.2 & 0.8 & 1.8 & 111 & 7.3 & 1.7 & 5.6 \\
\hline L. Cyambwe & 1290 & 19.2 & 6.7 & 4.1 & 0.6 & 1.3 & 110 & 6.8 & 1.3 & 7.0 \\
\hline L. Ihema & 1290 & 90.0 & 7 & 4.5 & 0.5 & 1.1 & 99 & 7.9 & 1.0 & 5.4 \\
\hline
\end{tabular}

S. C. E. (\% total)

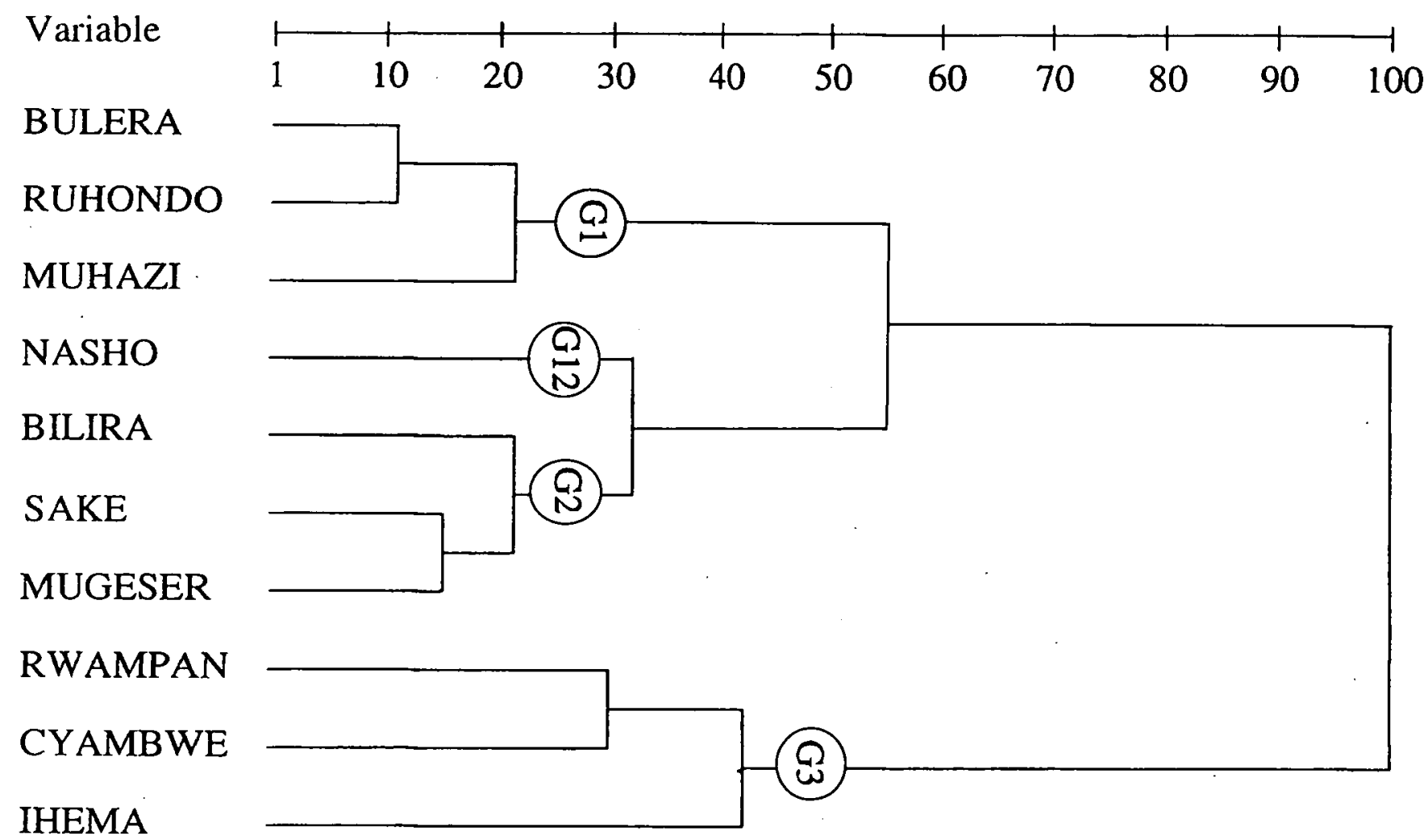

Fig. 2. Dendrogramme du «cluster analysis» sur les biomasses des poissons capturés. Classification des échantillons des lacs.

Fig. 2. Dendrogram classification of the lakes studied based on cluster analysis of fish biomass. 
Tableau 2. Captures totales des poissons dans les dix lacs rwandais étudiés.

Table 2. Total catch of fish in the ten Rwandan lakes.

\begin{tabular}{|c|c|c|c|c|c|c|c|c|c|c|}
\hline \multirow{2}{*}{\multicolumn{11}{|c|}{ CICHIIDAE }} \\
\hline & & & & & & & & & & \\
\hline Oreochromis niloticus (Linnaeus 1758) & 62 & 46 & 16 & 14 & 8 & 78 & 13 & 2 & 27 & 43 \\
\hline O. macrochir (Blgr 1912) & 9 & 3 & 8 & 30 & 5 & 97 & 88 & 196 & 54 & 0 \\
\hline O. leucostictus (Trewawas 1933) & $\mathbf{0}$ & 0 & 0 & 0 & 0 & 7 & 0 & 0 & 3 & 0 \\
\hline O. macrochir macrochir (Blgr 1912) & 0 & 0 & 0 & 0 & 0 & 0 & 0 & 0 & 38 & 0 \\
\hline Tilapia rendalli (Blgr 1896) & 0 & 0 & 2 & 1 & 2 & 3 & 2 & 2 & 1 & 5 \\
\hline Haplochromis spp. & 61 & 204 & 229 & 89 & 13 & 194 & 177 & 150 & 211 & 190 \\
\hline H. burtoni (Günther 1893) & 2 & 1 & 0 & 0 & 0 & 0 & 0 & 0 & 0 & 8 \\
\hline Paralabidochromis victoriae (Greenwood 1956) & $\mathbf{0}$ & 0 & 8 & 4 & 0 & 3 & 23 & 4 & 19 & 0 \\
\hline Astatoreochromis alluaudi (Pellegrin 1904) & 0 & 0 & 0 & 1 & 0 & 0 & 4 & 3 & 0 & 0 \\
\hline \multicolumn{11}{|l|}{ CYPRINIDAE } \\
\hline Barbus altianalis (Blgr 1900) & 0 & 0 & 0 & 2 & 0 & 4 & 1 & 1 & 0 & 0 \\
\hline B. paludinosus (Peters 1852) & 0 & 0 & 0 & 0 & 0 & 23 & 5 & 0 & 0 & 3 \\
\hline B. acuticeps (Matthes 1954) & 0 & 0 & 0 & 2 & 0 & 1 & 0 & 0 & 0 & 0 \\
\hline Barbus sp. & 0 & 0 & 0 & 0 & 0 & 70 & 0 & 0 & 8 & 0 \\
\hline Labeo victorianus (Blgr 1901) & 0 & 0 & 31 & 124 & 27 & 101 & 16 & 0 & 1 & 0 \\
\hline \multicolumn{11}{|l|}{ CLARIIDAE } \\
\hline Clarias gariepinus (Burchell 1822) & 0 & 0 & 39 & 17 & 31 & 38 & 54 & 56 & 17 & 11 \\
\hline C. liocephalus (Blgr 1898) & 7 & 0 & 0 & 0 & 0 & 0 & 0 & 0 & 0 & 0 \\
\hline \multicolumn{11}{|l|}{ MORMYRIDAE } \\
\hline Mormyrus kannume (Forskal 1775) & 0 & 0 & 0 & 0 & 0 & 2 & 0 & 0 & 0 & 0 \\
\hline Gnathonemus longibarbis (Hilgendorf 1888) & 0 & 0 & 0 & 0 & 0 & 15 & 25 & 0 & 12 & 0 \\
\hline Marcusenius victoriae (Linne 1758) & 0 & 0 & 0 & 0 & 0 & 10 & 23 & 0 & 6 & 0 \\
\hline M. cyprinoides (Linne 1758) & 0 & 0 & 0 & 0 & 0 & 1 & 0 & 0 & 0 & 0 \\
\hline Hippopotamyrus grahami (Norman 1928) & 0 & 0 & 0 & 0 & 0 & 43 & 42 & 0 & 0 & 0 \\
\hline Pollimyrus nigricans (Blgr 1906) & 0 & 0 & 1 & 0 & 0 & 0 & 1 & 0 & 0 & 0 \\
\hline \multicolumn{11}{|l|}{ MOCHOKIDAE } \\
\hline Synodontis afrofischeri (Hilgendorf 1888) & 0 & 0 & 0 & 0 & 0 & 10 & 51 & 1 & 27 & 0 \\
\hline S. ruandae (Matthes 1959) & 0 & 0 & 2 & 2 & 0 & 5 & 11 & 1 & 0 & 0 \\
\hline S. victoriae (Blgr 1906) & 0 & 0 & 0 & 0 & 0 & 4 & 4 & 0 & 0 & 0 \\
\hline \multicolumn{11}{|l|}{ CHARACIDAE } \\
\hline Alestes sp. & 0 & 0 & 0 & 0 & 0 & 15 & 11 & 0 & 0 & 0 \\
\hline A. nurse (Ruppell 1822) & $\mathbf{0}$ & 0 & 0 & 0 & 0 & 8 & 12 & 0 & 0 & 0 \\
\hline A. jacksonii (Blgr 1912) & 0 & 0 & 0 & 0 & 0 & 75 & 38 & 0 & 130 & 0 \\
\hline \multicolumn{11}{|l|}{ SCHILBEIDAE } \\
\hline Schilbe mystus (Linnaeus 1758) & 0 & 0 & 55 & 13 & 3 & 79 & 10 & 0 & 64 & 0 \\
\hline \multicolumn{11}{|l|}{ MASTACEMBELIDAE } \\
\hline Afromastacembelus frenatus (Blgr 1901) & 0 & 0 & 5 & 2 & 0 & 0 & 0 & 4 & 0 & 0 \\
\hline Biomasse capturée $\left(k_{\varepsilon}\right.$ & 141 & 254 & 396 & 301 & 89 & 886 & 611 & 420 & 618 & 260 \\
\hline Nombre de taxons rencontrés & $\mathbf{5}$ & 4 & 11 & 13 & 7 & 24 & 21 & 11 & 15 & 6 \\
\hline
\end{tabular}

Ce regroupement correspond à la distribution des lacs dans le plan des deux premières composantes exprimant respectivement 60 et $13 \%$ de la variabilité (Fig. 3). En effet les résultats de 1'ACP montrent l'existence de trois groupes distincts : le groupe Bulera, Ruhondo, Muhazi, le groupe Mugesera, Sake, Bilira et le groupe Rwampanga, Cyambwe, Ihema. Le lac Nasho, de la plaine de l'Akagera, est intermédiaire entre les lacs du Bugesera et ceux de l'Akagera.
La distribution des taxons de poissons dans le plan des deux premières composantes principales (Fig. 4) présente une sorte de continuum sur une structure curvilinéaire représentant de moins en moins de taxons suivant le sens des aiguilles d'une montre. Le nombre de taxons augmente des lacs Bulera et Ruhondo aux lacs Rwampanga , Ihema et Cyambwe. Le classement des taxons obtenus le long de cette structure curvilinéaire est reporté en abscisse de la figure 5. 


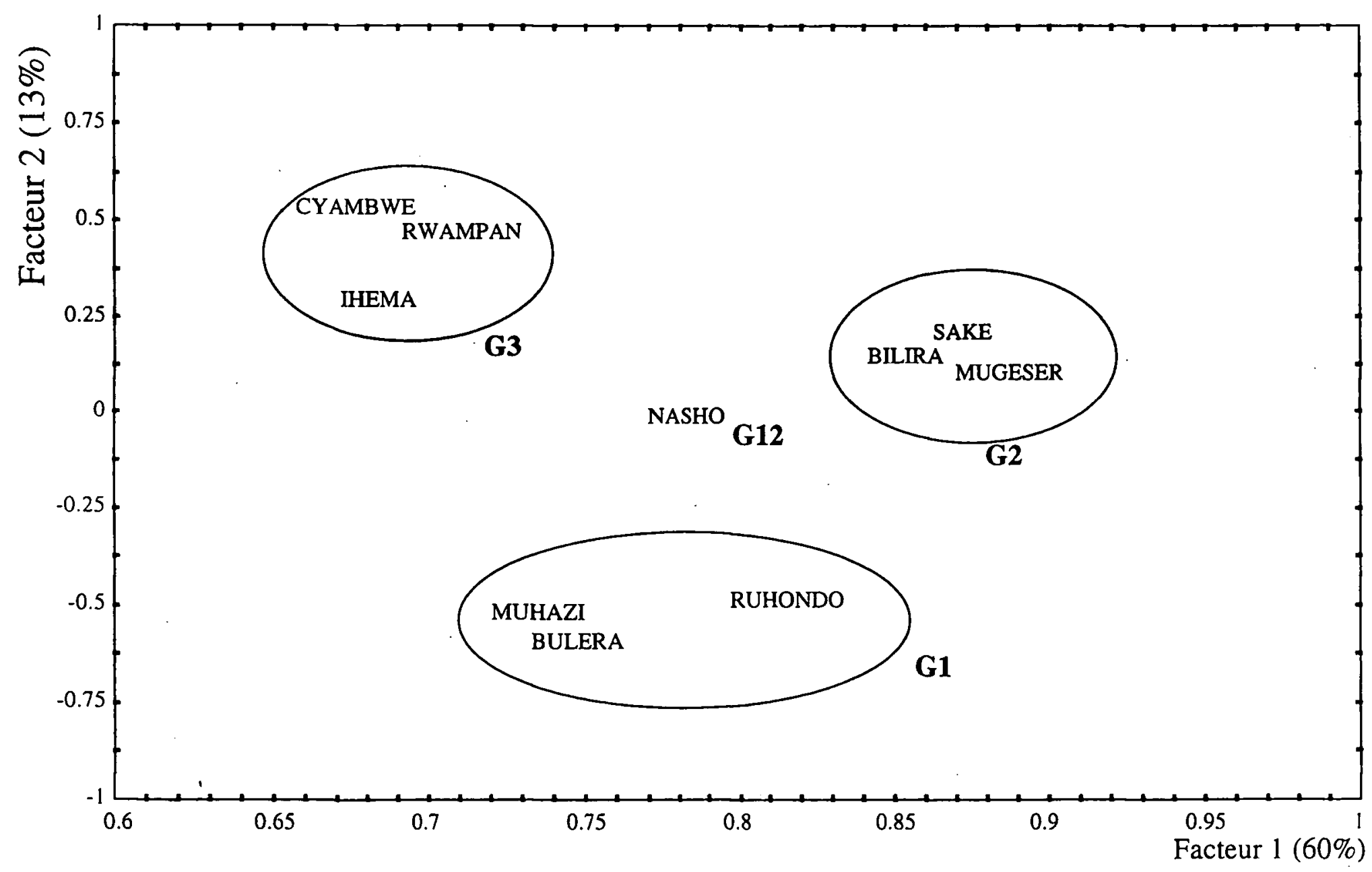

Fig. 3. Représentation des dix lacs rwandais étudiés dans le plan des deux premières composantes principales.

Fig. 3. Grouping of the ten Rwandan lakes studied based on the two first principal components.

Ce diagramme montre que les lacs Bulera et Ruhondo ne sont peuplés respectivement que de 5 et 4 taxons de poissons. Leur faune piscicole est fort peu diversifiée et dominée par les petits Cichlidés du genre $\mathrm{Ha}$ plochromis, suivis d'Oreochromis niloticus. Le lac Muhazi, placé dans le même groupe, est peuplé de 6 taxons dont le plus important en biomasse est celui des Cichlidés du genre Haplochromis. Ce lac a également une faune peu diversifiée et peu abondante.

Les lacs Mugesera, Sake et Bilira, de la dépression du Bugesera, présentent une faune piscicole relativement plus abondante et plus variée - de 7 à 13 taxons-. Elle est dominée par Haplochromis sp. mais voit la proportion d'O. niloticus, $O$. macrochir, Labeo victorianus, Clarias gariepinus et de Schilbe mystus augmenter sensiblement. Le lac Nasho, de la plaine de l'Akagera, proche de ce groupe, est peuplé de 11 taxons dont $O$. macrochir, Haplochromis sp. et $C$. gariepinus sont les plus importants.

Le dernier groupe comprend les lacs Rwampanga, Cyambwe et Ihema de la plaine de l'Akagera. Ces lacs ont une faune plus variée - de 15 à 24 taxons -, plus abondante et dont différentes espèces sont bien représentées. Ainsi, bien que Haplochromis sp. reste le taxon le plus abondant, les genres Oreochromis, Labeo, Clarias ( $C$. gariepinus), Alestes et Schilbe sont très bien représentés.

\section{Discussion et conclusion}

Les résultats d'analyse des données de peuplements de poissons révèlent une structure continue en croissant, caractéristique d'un continuum (Depiereux et al. 1983, 1985). Les lacs rwandais se présentent comme des stations le long d'un gradient amont-aval du complexe Nyabarongo-Akagera (Fig. 1) selon lequel la faune piscicole se diversifie et devient de plus en plus abondante. En effet seuls 4 taxons sont capturés dans les lacs Bulera et Ruhondo, en tête de bassin, 6 au lac Muhazi, de 7 à 13 dans les lacs du Bugesera et de 20 à 24 dans les lacs de l'Akagera, à l'est du pays. Le nombre et le poids de poissons capturés augmentent également d'amont en aval (Tableau 2). Ce constat est aussi celui de Micha \& Frank (1992). 


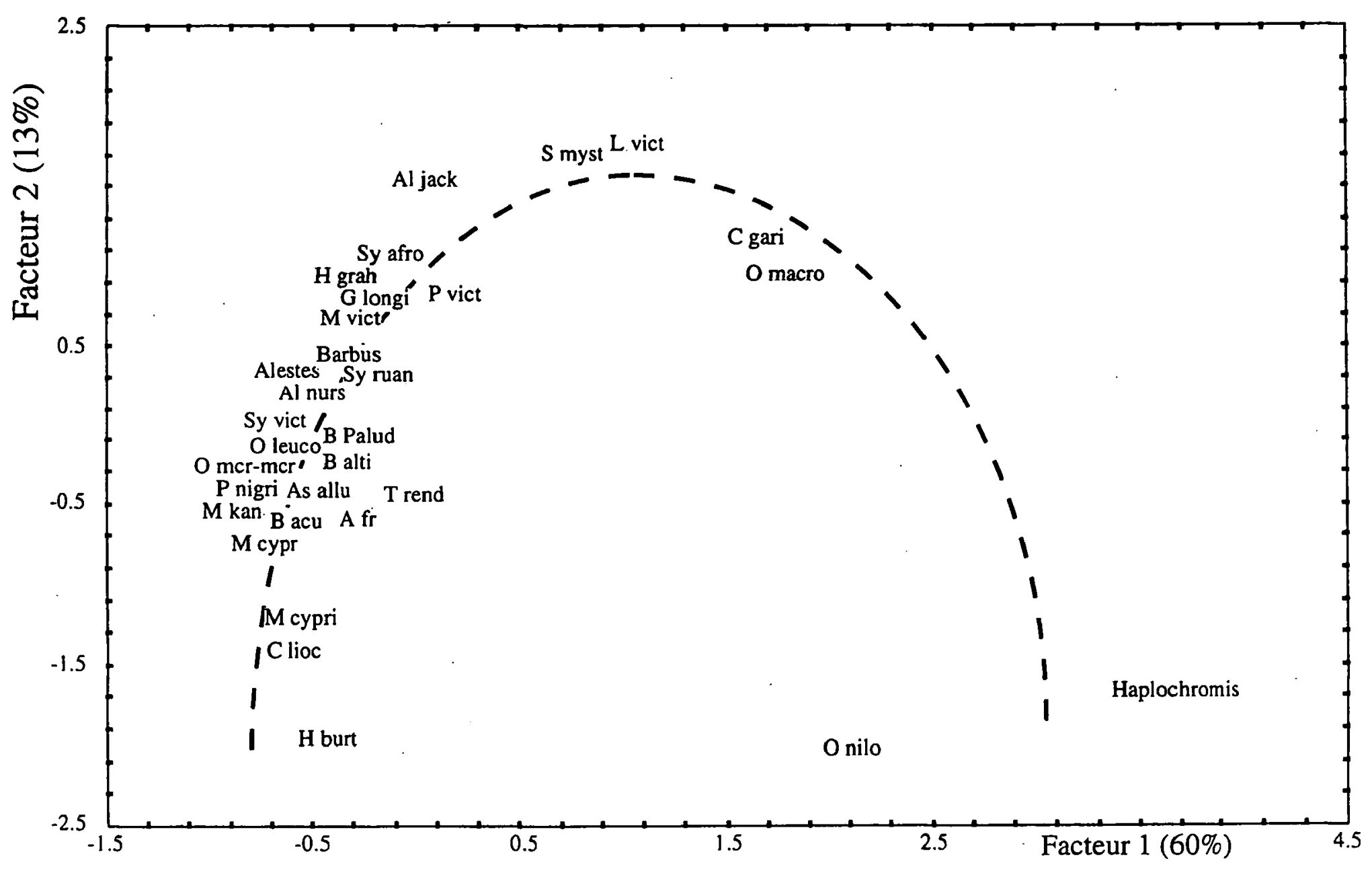

Fig. 4. Représentation des taxons de poissons des dix lacs rwandais étudiés dans le plan des deux premières composantes principales.

Fig. 4. Grouping of fish taxa in the ten Rwandan lakes studied based on the two first principal components.

Ce phénomène courant en milieu lotique expliquerait l'importance du rôle joué par le réseau fluviatile principal Nyabarongo-Akagera dans la biodiversité des écosystèmes lentiques rwandais. En effet, l'augmentation vers l'aval de la richesse taxonomique et de la productivité piscicole le long de ce réseau s'expliquerait par la présence des chutes et rapides (cas de la chute de Rusumo) qui constituent des barrières de plus en plus nombreuses s'opposant à la migration anadrome des poissons du lac Victoria vers les lacs de la Haute Akagera, et de là vers ceux des Volcans, au nord du pays. En d'autres termes, la colonisation par diverses espèces de poissons du complexe amont de la Nyabarongo a été fortement réduite par ces barrières physiques, ce qui explique leur faible biodiversité.

Par ailleurs, la communication plus ou moins étroite entre certains lacs et la rivière Akagera constituerait également un facteur favorisant la diversité taxonomique des poissons. Tel serait le cas des lacs Rwampanga, Cyambwe et Ihema bien diversifiés et bien poissonneux. Pour beaucoup d'autres lacs, particuliè- rement ceux de la dépression du Bugesera (Mugesera, Sake et Bilira, Fig.1), le marais de papyrus qui les sépare de la rivière Nyabarongo constituerait un filtre empêchant l'accès à ces lacs, de certaines espèces de poissons (Tableau 2) telles que Barbus paludinosus, Gnathonemus longibarbis, Marcusenius victoriae, Alestes jacksoni, etc..).

Il faut également signaler la biomasse remarquable des petits Cichlidés du genre Haplochromis qui occupe $40,2 \%$ des captures expérimentales totales des lacs rwandais considérés. Cette proportion atteint $80,7 \%$ au lac Ruhondo et 76,2 \% au lac Muhazi. Cette biomasse remarquable insoupçonnée jusqu'alors résulte probablement de la faible biodiversité observée dans ces lacs et, par conséquent, de l'absence de compétition d'autres espèces de poissons.

D'une façon générale, les lacs rwandais étudiés présentent une très faible biodiversité de leurs peuplements de poissons par rapport aux autres lacs africains, y compris ceux du bassin du Nil. Cela résulte probablement d'une part de leur petite taille mais aussi et 


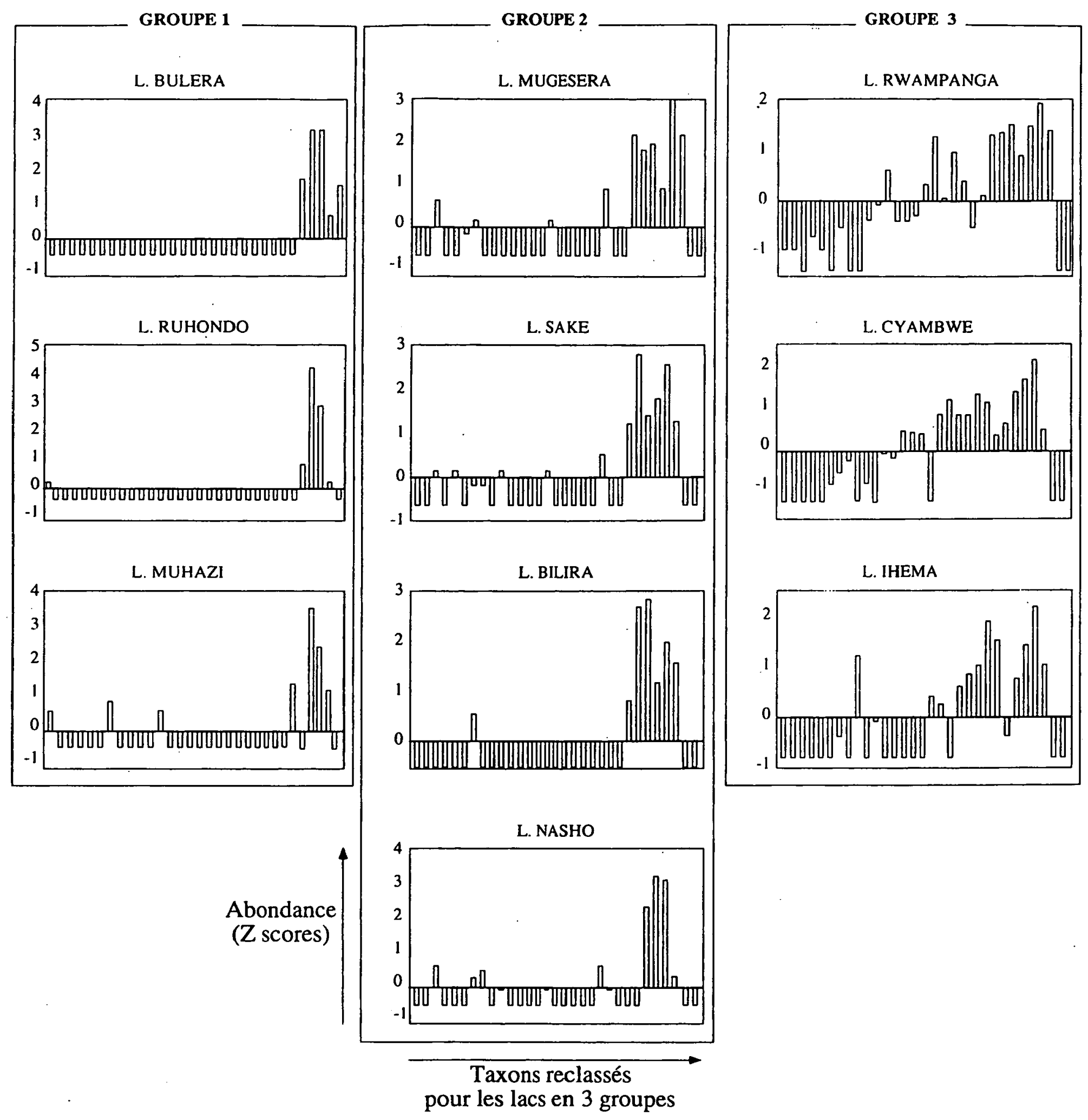

Fig. 5. Profil du peuplement ichtyologique des lacs étudiés, regroupés suivant la Fig. 3. En abscisse, les taxons ordonnés suivant le continuum (Fig. 4). En ordonnée, la biomasse standardisée (Z score).

Fig. 5. Ichthyological population profiles of the studied lakes, clustered as in Fig. 3. X-axis : taxa ordered following the continuum (Fig. 4). Y Axis : standardized biomass.

surtout des barrières physiques (chutes) qui ont empêché la colonisation anadrome du complexe de la Nyabarongo. Ceci suppose l'existence de niches écologiques encore disponibles. A l'instar du lac Kivu où l'introduction raisonnée et réussie d'un Clupéide du lac Tanganyika, Limnothrissa miodon Blgr, a permis d'augmenter les captures de poisson de plusieurs tonnes par an (Kaningini 1994), diverses introductions devraient être prudemment envisagées. Toutefois, dans chaque cas, une étude hydrobiologique approfondie de 
ces lacs est nécessaire pour bien mettre en évidence les niches écologiques vacantes et envisager de les combler par des introductions d'espèces adéquates présentes dans le bassin du Nil. Il ne pourrait être question de répéter le cas du lac Victoria où l'introduction accidentelle du Lates niloticus qui bien que commercialement devenu très important a bouleversé complètement la biodiversité remarquable de ce lac.

La position du lac Muhazi, lac eutrophe, parmi les lacs d'altitude à faible productivité primaire et piscicole mérite à ce propos une considération particulière. En effet, le lac Muhazi, l'un des mieux connus, malgré une flore planctonique riche et dense, une faune d'invertébrés fort abondante et riche en mollusques (Damas 1956, Phillipot 1987) présente une faune piscicole fort peu diversifiée. Des niches écologiques y sont encore disponibles. En plus des herbivores, les malacophages pourraient efficacement contribuer à l'augmentation de la production piscicole de ce lac. C'est pour valoriser le stock de mollusques et occuper ainsi une des niches disponibles que Protopterus aethiopicus (Heckel) a été introduit dans le lac Muhazi en 1990 (Mukankomeje et al. 1996). Il faudrait maintenant faire des pêches expérimentales pour évaluer le stock, former les pêcheurs locaux aux techniques particulières à la capture de cette espèce et passer ensuite à une phase d'exploitation durable.

La caractérisation de nos dix lacs rwandais basée sur les peuplements de poissons est différente de celle basée sur les données physiques et chimiques (cfr. $\S$ Données physiques et chimiques) et phytoplanctoniques (Descy 1995). La première distingue essentiellement deux grands groupes de lacs : profonds et peu profonds. La dernière met en évidence, par la similitude de leurs communautés algales, un grand groupe compact formé des lacs de la dépression du Bugesera et ceux de l'Akagera, à côté du groupe Bulera, Ruhondo et des cas exceptionnels comme les lacs Rwampanga et Nasho, au phytoplancton dense et varié. Il est vrai que les caratéristiques physiques et chimiques des lacs et leurs peuplements algaux sont beaucoup moins sensibles aux barrières physiques (chutes) que les poissons. Ceci explique probablement cela!

Cependant il reste encore beaucoup à faire pour bien caractériser tous les lacs rwandais (environ 20 lacs), appartenant à deux bassins versants (Nil et Congo), sur base de leur faune ichtyologique. Un inventaire taxonomique régulier des lacs et de la rivière NyabarongoAkagera (bassin du Nil) ainsi que du complexe de la Ruzizi (bassin du Congo) et une meilleure détermination des espèces telles que celles du genre Haplochro- mis ainsi qu'une meilleure évaluation de leurs biomasses sont encore nécessaires et devraient faire l'objet d'un programme coordonné.

\section{Travaux cités}

BUNEP 1988. - Bureau National d'Etudes et Programmes. Etude pour le développement de la pêche et l'empoissonnement des lacs du nord et de l'est du Rwanda. Rapport définitif. Phase II. Ministère de l'Agriculture et de l'Elevage (Rwanda) : $434 \mathrm{p}$.

Damas H. 1953. - Les lacs du Ruanda et leurs problèmes. Ann. Soc. Roy. Zool. Belg., 84 : 17-38.

Damas H. 1954 a. - Etude limnologique de quelques lacs ruandais. I. Le cadre géographique. Mém. Inst. Royal Colon. Belge, $\mathrm{Cl}$. Sci. nat. et méd., nouv. sér. in- $8^{\circ}, 24$ (2) : $92 \mathrm{p}+$ annexes.

Damas H. 1954 b. - Etude limnologique de quelques lacs ruandais. II. Etude thermique et chimique. Mém. Inst. Royal Colon. Belge, Cl. Sci. nat. et méd., nouv. sér., 24 (4) : 116 p.

Damas H. 1955. - Etude limnologique de quelques lacs rwandais. III. Le plancton. Mém. Inst. Royal Colon. Belge, Cl. Sci. nat. et méd., nouv. sér., 1 (3) : 66 p.

Damas H. 1956. - Etude limnologique de quelques lacs rwandais. IV. Les sédiments et leur faune. Conclusions générales. Mém. Inst. Royal Colon. Belge, $\mathrm{Cl}$. Sci. nat. et méd., nouv. sér. in $-8^{\circ}$, $3(4): 47 \mathrm{p}$.

Depiereux E., Feytmans E. \& Micha J.-C. 1983. - Utilisation critique de l'analyse en composantes principales et du cluster analysis pour la description d'échantillons d'invertébrés benthiques en eau douce. Oikos, $40: 81-94$.

Depiereux E. \& Feytmans E. 1985. - Modification progressive de la structure des peuplements d'invertébrés benthiques en fonction de la qualité de l'eau de l'Ourthe et de la Lesse (Meuse belge). Acta oecol. / Oecol. applicata, 6 (2) : 81-98.

Descy J.-P., 1995. - Essai de caractérisation des lacs du Rwanda par leur communauté phytoplanctonique. Acad. Royale des Sci d'Outre-Mer : 15-31.

Kanangire K. 1988. - Typologie des lacs rwandais sur base de leurs caracteristiques abiotiques et biotiques. Mém. Certif. Ecologie des Eaux Continentales, FUNDP, Namur : 81 p. + annexes.

Kaningini B. 1994. - Etude de la croissance, de la reproduction et de l'exploitation de Limnothrissa miodon au lac Kivu, Bassin de Bukavu (Zaïre). Presses Universitaires de Namur, Namur : $168 \mathrm{p}$.

Kiss R. 1976. - Etude hydrobiologique des lacs de l'Akagera moyenne, Institut de Recherche Scientifique de Butare. Publication $\mathrm{n}^{\circ} 16: 167 \mathrm{p}$.

Kiss R. 1977. - Les poissons et la pêche dans le lac Ihema (Rwanda, bassin moyen de l'Akagera), Ann. Hydrobiol., 17 (2) : 263-318.

Lebart L., Morineau A. \& Fenelon J.-P. 1979. - Traitement des données statistiques, méthodes et programmes. Bordas, Paris : $510 \mathrm{p}$.

Legendre L. \& Legendre P. 1979. - Ecologie numérique: Vol. 1 : Le traitement multiple des données écologiques, Masson, Paris. : 197 p. Vol. 2 : La structure des données écologiques, Masson, $\mathrm{Pa}-$ ris. : $254 \mathrm{p}$.

Micha J.-C. \& Frank V. 1992. - Ressources et caractéristiques piscicoles des lacs rwandais (Bassin nilotique). Bull. Séanc. Acad. $r$. Sci. Outre-Mer, 37 (3) : 379-398.

Mukankomeje R. 1992. - Production algale et consommation par le tilapia, Oreochromis niloticus L., au Lac Muhazi (Rwanda). Thèse de Doctorat, FUNDP, Namur, Belgique : $254 \mathrm{p}$.

Mukankomeje R., Plisnier P. D., Descy J.-P. \& Massaut L. 1993. Lake Muhazi, Rwanda : limnological features and phytoplankton production. Hydrobiologia, $257: 107-120$. 
Mukankomeje R., Micha J.-C., Descy J.-P. \& Frank V. 1996. — Production potentielle d'une espèce introduite Protopterus aethiopicus (Heckel 1871) basée sur la modélisation de l'écosystème du lac Muhazi (Rwanda). Bull. Séanc. Acad. r. Sci. Outre-Mer, 42 (3): 459-483.

Phillipot J.M. 1987. — Faune aquatique des mollusques du lac $\mathrm{Mu}-$ hazi (Rwanda) : Une contribution faunique destinée à l'étude des possibilités d'introduction de Protopterus aethiopicus (HECKEL) (Poisson Dipneuste) et de l'épidémiologie de la Bilharziose. Mém. Ing. Agron., Fac. sc. agronomiques, Gembloux : 103 p. + annexes.
Plisnier P. D. 1990. - Ecologie comparée et exploitation rationnelle de deux populations d'Haplochromis spp. (Teleostei, Cichlidae) des lacs Ihema et Muhazi (Rwanda). Thèse de Doctorat, UCL, Louvain-la-Neuve, Belgique : 328 p.

Plisnier P. D., Micha J.-C. \& Frank V. 1988. —Biologie et exploitation des poissons du lac Ihema (Bassin Akagera, Rwanda). Presses Universitaires de Namur, Namur : 212 p.

Statsoft 1994. - Statistica. Quick reference, Statsoft Technical Support : 149 p. 